\title{
Bemerkung über die von Dirichlet in seiner Breslauer Habilitationsschrift behandelten Functionen.
}

\author{
Von R. Daublebsky yon Sterneck in Wien.
}

Lejeune-Dirichlet hat sich in seiner Breslauer Habilitationsschrift mit den Functionen beschäftigt, welche entstehen, wenn in dem Ausdrucke $(x+\sqrt{b})^{n}(x, b, n$ ganze Zahlen, $b$ keine Quadratzahl) der rationale und irrationale Bestandtheil getrennt wird. ${ }^{1}$ ) Namentlich untersucht er den Coefficienten ron $\sqrt{b}$ bezüglich seiner Zerlegung in Primfactoren für jeden beliebjgen Exponenten $n$.

In einer vor kurzem in diesen "Monatsheften " ") erschienenen Mittheilung habe ich bezüglich einiger, durch specielle Eigenschaften charakterisierter zahlentheoretischer Functionen ein allgemeines Theorem abgeleitet, welches über die primitiven (nicht in $f(x), x<n$, aufgehenden) Primfactoren von $f(n)$ Aufschluss gibt, und als Beispiel eine Function betrachtet, welche sich als einfache Verallgemeinerung aus dem allgemeinen Gliede der L am éschen Reihe ergab, ihrerseits aber wieder ein specieller Fall jener von Dirichle untersuchten Functionen ist. In den folgenden Zeilen möchte ich nun, als Nachtrag zu jener Note, zeigen, dass auch die allgemeineren Dirichlet'schen Functionen selbst unter denselben Gesichtspunkt fallen, dass sich von denselben ebenfalls die erwähnten charakteristischen Eigenschaften nachweisen lassen, und somit auch die Giltigkeit des Theoremes auf diese Functionen ausgedehnt werden kann; ferner wird sich ein, von Lucas gegebenes, Primzahlkriterium, welches sich auf gewisse Eigenschaften der Lamé schen Reihe gründet, ebenfalls leicht auf diese allgemeineren Functionen iibertragen lassen.

1.

Es sei $f(n)$ dureh die Recursionsformel:

(1) $f(n)=\alpha f(n-1)+\beta f(n-2) ; \quad(\alpha, \beta$ theilerfremde ganze Zahien) und die Anfangsbedingungen $f(1)=1, f(2)=\alpha$ definiert.

1) Lejeune-Dirichlet's Werke. Hg. von Kronecker. Bd. 1, s. $4 \%$. Kronecker, Sitrungsber. der k. preuf. Akad. Jahrg. 1888.

2) VII. Jahrg. S. 37. 
Dann genügt $f(n)$ auch der weiteren Recursionsformel:

$$
f(n)=f(\lambda) f(n-(\lambda-1))+\beta f(\lambda-1) f(n-\lambda),
$$

was sich durch den Schluss von $\lambda$ auf $\lambda+1$ leicht nachweisen lässt.

Als independenten Ausdruck für $f(n)$ erhält man, wenn man $\alpha^{2}+4 \beta=P$ setzt:

$$
\begin{gathered}
f(n)=\frac{1}{2^{n-1}}\left\{\left(\begin{array}{l}
n \\
1
\end{array}\right) \alpha^{n-1}+\left(\begin{array}{l}
n \\
3
\end{array}\right) \alpha^{n-3} P+\left(\begin{array}{l}
n \\
5
\end{array}\right) \alpha^{n-5} P^{2}+\cdots\right\}= \\
=\frac{1}{\sqrt{P}}\left\{\left(\frac{\alpha+\sqrt{P}}{2}\right)^{n}-\left(\frac{\alpha-\sqrt{P}}{2}\right)^{n}\right\} .
\end{gathered}
$$

Es handelt sich nun darum, von der Function $f(n)$ die vier, in der erwähnten Note mit $(a),(b),(c),(d)$ bezeichneten Eigenschaften nachzuweisen:

a) $f(1)=1$; ist erfiillt.

b) $g[f(m), f(n)]=f[g(m, n)]$, wo $g$ den größten gemeinsamen Theiler bedeutet.

Weil $\alpha$ und $\beta$ theilerfremd vorausgesetzt sind, kann zunächst auf Grund ron (1) für kein Argument $n, f(n)$ mit $\beta$ einen Theiler gemein haben, denn dieser Theiler müsste $f(n-1)$, analog $f(n-2), \ldots$ endlich $f(1)$ theilen.

Daher können für kein Argument $n, f(n)$ und $f(n-1)$ einen Theiler gemein haben; denn dieser, weil nach der eben gemachten Bemerkung zu $\beta$ theilerfremd, müsste $f(n-2), f(n-3)$, endlich $f(1)$ theilen.

Aus (2) folgt somit, dass jeder gem. Theiler von $f(n)$ und $f(\lambda)$ Theiler von $f(n-\lambda)$ sein muss; daraus ergibt sich, dass $g(f(m), f(n))$ in $f(g(m, n))$ aufgeht; andererseits lehrt die Formel (2), wenn man successive $\lambda=2,3, \ldots$ setzt, dass sowohl $f(m)$ als $f(n)$ durch $f(g(m, n))$ theilbar ist; also muss $g(f(m), f(n))$ mit $f(g(m, n))$ identisch sein.

c) Für jede Primzahl $p$, bis auf eine endliche Anzahl von Ausnahmen, ist $f(p+1)$ oder $f(p-1) \equiv 0$ (mod. $p$ ). Aus (3) ergibt sich zunächst, wenn $p$ eine Primzahl ist, die weder $2 \alpha$ noch $P$ theilt:

$$
2^{p} f(p+1) \equiv \alpha^{p}+\left(\begin{array}{c}
p+1 \\
p
\end{array}\right) \alpha P^{\frac{p-1}{2}} \equiv \alpha\left(1+P^{\frac{p-1}{2}}\right)(\bmod \cdot p),
$$

also: $f(p+1) \equiv 0(\bmod . p)$, wenn $P$ quadratischer Nichtrest von $p$ ist, und $f(p+1) \equiv \alpha$ (mod. $p)$, wenn $P$ quadratischer Rest von $p$ ist; in letzterem Falle findet man aber aus $(3) f(p) \equiv 1$ (mod. $p)$; somit folgt aus der Recursionsformel (1) $f(p-1) \equiv 0(\bmod . p)$. 
d) Der Quotient $\frac{f(\rho n)}{f(n)}$ hat mit $f(n)$ höchstens einen Theiler von $?$ gemein; dies folgt aus der Recursionsformel (2) durch öftere Anwendung. ${ }^{1}$ )

Da somit die vier charakteristischen Eigenschaften $(a),(b)$, $(c),(d)$ erfüllt sind, gilt das eingangs erwähnte Theorem ${ }^{2}$ ) auch bezüglich dieser Functionen $f(n)$.

Die von Dirichlet behandelten Functionen genügen aber in der That der Recursionsformel (1); denn setzt man $\alpha=2 x$, $\beta=b-x^{2}$, so wird $P=4 b$ und man erhält

$$
f(n)=\frac{1}{\sqrt{P}}\left\{\left(\frac{2 x+\sqrt{P}}{2}\right)^{n}-\left(\frac{2 x-\sqrt{P}}{2}\right)^{n}\right\}=\frac{(x+\sqrt{b})^{n}-(x-\sqrt{b})^{n}}{2 \sqrt{b}},
$$

so dass $f(n)$ in der That mit dem Coefficienten von $\sqrt{b}$ bei der Trennung von $(x+\sqrt{b})^{n}$ in den rationalen und irrationalen Bestandtheil identisch wird. Die Anfangsbedingungen $f(1)=1$, $f(2)=\alpha=2 x$ sind ebenfalls erfüllt.

2.

Das von $\mathrm{Lucas}^{3}$ ) aufgestellte Primzahlcriterium lautet: Is $t$ $f(x)$ das $*^{\text {te }}$ Glied der Lamé'sehen Reihe und ist $x=n+1$ oder $x=n-1$ das kleinste Argument, für welches $f(x)$ durch $n$ theilbar wird, so ist $n$ eine Primzahl.

Es soll nun gezeigt werden, dass dasselbe Criterium auch giltig bleibt, wenn $f(x)$ die obige allgemeinere Function bezeichnet.

Dies lässt sich sehr einfach beweisen.

Setzen wir zur Abkürzung:

$$
\frac{\alpha+\sqrt{P}}{2}=A ; \quad \frac{\alpha-\sqrt{P}}{2}=B ; \quad \sqrt{P} f(n)=A^{n}-B^{n} .
$$

Nehmen wir an, es sei $p$ eine Primzahl, und $x$ das kleinste Argument, für welches

$$
f(x) \equiv 0\left(\bmod \cdot p^{i}\right)
$$

wird; es lässt sich dann auch unmittelbar das kleinste Argumant $x^{\prime}$ angeben, für welches

$$
f\left(x^{\prime}\right) \equiv 0\left(\bmod \cdot p^{i+1}\right)
$$

1) Monatshefte, VII. Jahrg., S. 43.

2) Monatshefte, VII. Jahrg., S. 37.

3) Bullettino di bibliografia $e$ di storia delle scienze matematiche e fisich $\theta$ da B. Boncompagni. Tomo X, pag. 153. Roma 1877. 
wird. Weil nämlich $x$ das kleinste Argument ist, so folgt aus der Eigenschaft $(b)$, dass $x^{\prime}$ jedenfalls ein Vielfaches von $x$ sein muss, andererseits folgt aus der Beziehung:

$$
\begin{aligned}
{[f(x)]^{p} P^{\frac{p}{2}}=A^{2 p}-B^{2 p}-\left(\begin{array}{l}
p \\
1
\end{array}\right) } & A B\left(A^{2(p-2)}-B^{x(p-2)}\right)+\cdots \\
& \pm\left(\begin{array}{c}
p \\
\frac{p-1}{2}
\end{array}\right) A^{\frac{p-1}{2}} B^{\frac{p-1}{2}}\left(A^{*}-B^{*}\right)
\end{aligned}
$$

was auch in der Form:

$$
[f(x)]^{p} P^{\frac{p-1}{2}}=f(x p)-\left(\begin{array}{l}
p \\
1
\end{array}\right) A B f(x(p-2))+\cdots \pm\left(\begin{array}{c}
p \\
\frac{p-1}{2}
\end{array}\right)(A B)^{\frac{p-1}{2}} f(x)
$$

geschrieben werden kann, unmittelbar

$$
f(x p) \equiv 0\left(\bmod \cdot p^{i+1}\right)
$$

so dass sich in eindeutiger Weise $x^{\prime}=x p$ ergibt.

Nebmen wir nun an, $n$ wäre zusammengesetzt:

$$
n=p_{1}^{\pi} p_{2}^{\pi_{2}} \ldots p_{r}^{\pi_{r}} \text {. }
$$

Da es der Voraussetzung nach ein Argument, nämlich $n \pm 1$ gibt, für welches $f$ durch $n$ theilbar wird, so muss es auch bezüglich jedes Theilers von $n$ ein kleinstes Argument geben, für welches $f$ durch den betreffenden Theiler von $n$ theilbar wird; alle diese Argumente müssen auf Grund der Eigenschaft $(b)$ Theiler von $n \pm 1$, also sämmtlich zu $n$ theilerfremd sein.

Es gibt also namentlich ein kleinstes Argument $a_{i}$, für welches $f\left(a_{i}\right) \equiv 0$ (mod. $p_{i}$ ) ist, und ebenso ein kleinstes Argument $a_{i}^{\prime}$, für welches $f\left(a_{i}^{\prime}\right) \equiv 0$ (mod. $\left.p_{i}^{\pi_{i}}\right)$ ist; es ist nun klar, dass $a_{i}=a_{i}^{\prime}$ sein muss; denn sonst mitsste nach dem eben bewiesenen Hilfssatze $a_{i}^{\prime}$ den Factor $p_{i}$, mindestens in der 1. Potenz, enthalten, während es, wie bemerkt wurde, zu $n$ theilerfremd sein muss.

$a_{i}$ ist aber ein Theiler von $p_{i} \pm 1$ (auf Grund der Eigenschaften $(b)$ und $(c)$ ); also gibt es für $i=1,2, \ldots, r$ Zahlen $a_{i}$, derart, dass $f\left(a_{i}\right) \equiv 0\left(\bmod . p_{i}^{\pi_{i}}\right)$ ist und andererseits $p_{i} \pm 1 \equiv 0$ $\left(\bmod . a_{i}\right)$ ist.

Das kleinste gem. Vielfache aller $a_{i}(i=1,2, \ldots, r)$ ist offenbar das kleinste Argument $x$, für welches $f(x)$ durch $n$ theilbar wird. Ist, wie vorausgesetzt ist, $x=n \pm 1$, so kann $n$ erstens keine Primzahlpotenz sein, deren Exponent größer als 1 ist (denn 
wenn $n=p_{1}^{\pi_{4}}, \pi_{1}>1$ wäre, so wäre der Voraussetzung nach $a_{1}=p_{1}^{\tau_{1}} \pm 1$, also gewiss kein Theiler von $p_{1} \pm 1$ ); $n$ kann aber auch nicht aus mehr als einer Primzahlpotenz bestehen, denn das kleinste gemeinsame Vielfache der $a_{i}$ ist gewiss nicht größer als das kleinste gemeinsame Vielfache der Zahlen $p_{i} \pm 1$, und da die letzteren lauter gerade Zahlen sind, gewiss

$$
\leqq \frac{1}{2^{r-1}} \prod_{i}\left(p_{i} \perp 1\right)
$$

woraus unmittelbar einleuchtet, dass dieses kleinste gemeinsame Vielfache für alle $r>1$ kleiner als $n-1$ ausfällt. Also muss $n$ eine Primzabl sein.

Selbstverständlich muss von der Zahl $n$, will man dieses Kriterium anwenden, bereits feststehen, dass sie durch keine jener Primzahlen theilbar ist, welche die Bedingung (c) nicht erfüllen. 